\title{
Prognostic Implications of Total Hemispheric Glucose Metabolism Ratio in Cerebrocerebellar Diaschisis
}

\author{
Eivind Antonsen Segtnan ${ }^{1,2}$, Peter Grupe ${ }^{1}$, Jens Ole Jarden ${ }^{3}$, Oke Gerke ${ }^{1,4}$, Jana Ivanidze ${ }^{5}$, Sofie Bæk Christlieb ${ }^{1}$, \\ Caius Constantinescu ${ }^{1}$, John Erling Pedersen ${ }^{1}$, Sina Houshmand ${ }^{6}$, Søren Hess ${ }^{1,7,8}$, Mojtaba Zarei ${ }^{9}$, Albert Gjedde ${ }^{2,10}$, \\ Abass Alavi ${ }^{6}$, and Poul F. Høilund-Carlsen ${ }^{1,8}$
}

\begin{abstract}
${ }^{I}$ Department of Nuclear Medicine, Odense University Hospital, Odense, Denmark; ${ }^{2}$ University of Southern Denmark, Odense, Denmark; ${ }^{3}$ Department of Neurology, Herlev University Hospital, Copenhagen, Denmark; ${ }^{4}$ Centre of Health Economics Research, Odense University of Southern Denmark, Odense, Denmark; ${ }^{5}$ Department of Diagnostic Radiology, Weill Cornell Medical College, NewYork-Presbyterian Hospital, New York, New York; ${ }^{6}$ Division of Nuclear Medicine, Department of Radiology, Perelman School of Medicine, Hospital of the University of Pennsylvania, Philadelphia, Pennsylvania; ${ }^{7}$ Department of Radiology and Nuclear Medicine, Hospital of Southwest Jutland, Esbjerg, Denmark; ${ }^{8}$ Department of Clinical Research, Faculty of Health Sciences, University of Southern Denmark, Odense, Denmark; ${ }^{9}$ National Brain Mapping Centre, Shahid Beheshti University (Medical and General Campus), Tehran, Iran; and ${ }^{10}$ Department of Neuroscience and Pharmacology, Panum Institute, University of Copenhagen, Copenhagen, Denmark
\end{abstract}

Diaschisis denotes brain dysfunction remote from a focal brain lesion. We have quantified diaschisis and investigated its prognostic value in glioma. Methods: We compared $50{ }^{18} \mathrm{~F}-\mathrm{FDG}$ PET/CT studies collected prospectively from 14 patients with supratentorial glioma (5 men and 9 women; age range, 35-77 y) with 10 single scans from healthy controls (age range, 43-75 y). Dedicated 3-dimensional segmentation software was used to obtain total hemispheric glucose metabolic ratios (THGr) by dividing total hemispheric ${ }^{18} \mathrm{~F}-\mathrm{FDG}$ uptake in each diaschitic hemisphere - that is, the ipsilateral cerebral hemisphere $(\operatorname{THGr}(\mathrm{Ce}))$ and the contralateral cerebellar hemisphere ( $\mathrm{THGr}(\mathrm{Cb})$ ) — by its respective contralateral side. Receiver-operatingcharacteristic (ROC) analysis was performed to determine optimal cut-offs for combinations of $\mathrm{THGr}(\mathrm{Ce})$ and $\mathrm{THGr}(\mathrm{Cb})$. Two independent observers obtained data for reproducibility analysis, and THGr values were compared with qualitative assessment of diaschisis performed by a PET neuroimaging specialist. Results: Qualitative analysis confirmed cerebrocerebellar diaschisis in all glioblastoma PET studies performed within $1 \mathrm{y}$ of death. Healthy subjects had significantly higher $\operatorname{THGr}(\mathrm{Ce})$ values $(P=0.0007)$ and $\mathrm{THGr}$ (Cb) values $(P=0.02)$ than glioblastoma patients. ROC analysis yielded diaschisis thresholds of 0.62 for $\operatorname{THGr}(\mathrm{Ce})$ and 0.84 for THGr (Cb). Qualitative assessment demonstrated cerebral diaschisis in 16 of 17 (94\%) cases with $\mathrm{THGr}(\mathrm{Ce})$ below the determined threshold and cerebellar diaschisis in 25 of $26(96 \%)$ cases with $\mathrm{THGr}(\mathrm{Cb})$ below the determined threshold. When both $\operatorname{THGr}(\mathrm{Ce})$ and $\operatorname{THGr}(\mathrm{Cb})$ were below the ROC threshold, the combined diaschisis measures had a positive predictive value for survival below $1 \mathrm{y}$ of $100 \%$. When one parameter was below the threshold, it had a positive predictive value of $75 \%$, and when both parameters exceeded thresholds, the negative predictive value for survival above 1 y was $79 \%$. Median interrater variability was $3.3 \%$ and $5.9 \%$ for $\operatorname{THGr}(\mathrm{Ce})$ and $\operatorname{THGr}(\mathrm{Cb})$, respectively. Conclusion: The THGr measures demonstrated diaschisis in the cerebrum and cerebellum of patients with glioma. Combined cerebrocerebellar diaschisis ratios with ROC thresholds for both forebrain and hindbrain had high negative and positive predictive values for

Received Jul. 21, 2016; revision accepted Oct. 4, 2016.

For correspondence or reprints contact: Eivind Antonsen Segtnan, Sankt Knuds gade 20, stuen, 5000 Odense C, Denmark.

E-mail: eivind.antonsen.segtnan@gmail.com

Published online Oct. 27, 2016.

COPYRIGHT (c) 2017 by the Society of Nuclear Medicine and Molecular Imaging. survival for less than a year. The THGr method allows comparison of data obtained at different institutions and is now open for further validation in gliomas and other cerebral diseases.

Key Words: diaschisis; brain; cerebellum; glioma; quantification; FDG-PET/CT

J Nucl Med 2017; 58:768-773

DOI: $10.2967 /$ jnumed.116.180398

D iaschisis is an oft-forgotten neurologic phenomenon. Introduced in 1914 by von Monakow, the term diaschisis denotes a loss or change of function in regions of the brain remote from the underlying structural (often discrete) lesion due to neuronal connectivity (1). With the trillions of connections in the human brain (2), diaschisis is a complex entity that is largely unexplored, and conventional structural imaging with $\mathrm{CT}$ and MRI are inadequate. In a recent review, Carrera et al. suggested 4 subclassifications of diaschisis (3) that may be more appropriate for mechanistic imaging. PET/CT with ${ }^{18} \mathrm{~F}$-FDG has shown promising results of visual image assessment of diaschisis (4-9). Gliomas invade brain parenchyma and destroy healthy brain tissue, and lesions in turn damage other elements of the connectome as manifested by diaschisis. Here, we tested a novel method for detection and quantification of diaschisis with ${ }^{18}$ F-FDG images from PET/CT in patients with gliomas, and we assessed the prognostic value of the presence of diaschisis.

\section{MATERIALS AND METHODS}

${ }^{18} \mathrm{~F}-\mathrm{FDG}$ images of the brain were obtained with PET/CT in 14 patients ( 5 men, 9 women; mean age, $63 \mathrm{y}$; age range, 35-77 y) who were admitted to the Department of Neurosurgery, Odense University Hospital, with suspicion of cerebral malignancy. Diagnosis based on biopsy and MRI showed that 2 patients had diffuse astrocytoma and 12 had glioblastoma. The patients were followed throughout their treatment course for $1 \mathrm{y}$, or until death. The ${ }^{18} \mathrm{~F}$-FDG images were acquired prospectively at up to 6 time points: (1) before treatment, (2) after resection or biopsy, (3) after radiotherapy or chemotherapy or no 
treatment, and (4-6) during chemotherapy or no treatment. Prednisolone was used during the treatment course of the glioma patients due to clinical practice. Baseline demographic and clinical characteristics of the study population are detailed in Supplemental Table 1 (supplemental materials are available at http://jnm.snmjournals.org). For radiotherapy, either $34 \mathrm{~Gy} / 10$ fractions or $59.4 \mathrm{~Gy} / 33$ fractions were used. Temozolomide was the standard chemotherapy. The baseline scan was obtained a mean of $9 \mathrm{~d}$ (range, 3-13 d) after the baseline MR images. Follow-up scans were obtained 35 d (range, 13-133 d), $121 \mathrm{~d}$ (range, 56-263 d), $339 \mathrm{~d}$ (range, $278-396 \mathrm{~d}$ ), and $466 \mathrm{~d}$ (range, 368-536 d) after the diagnostic MRI. Tumor volumes were obtained on the basis of volume segmentation of gadolinium-enhanced T1-weighted images using 3-dimensional (3D) Slicer Software (version 4.5; http://www.slicer.org), an open-source segmentation software program $(10)$.

The control group consisted of 10 healthy volunteers ( 5 men, 5 women; mean age, $62.5 \mathrm{y}$; age range, 43-75 y) without neurologic disease, severe brain trauma, addiction, or cognitive deficits. These control subjects underwent a single PET/CT session with the same acquisition protocol as for the patients. All participants gave informed written consent to participate in the study, which was approved by the regional research ethics committee.

\section{PET/CT Data Acquisition}

Participants fasted for $6 \mathrm{~h}$ before undergoing imaging. They rested supine on the tomograph bed in a darkened and quiet room, with heads immobilized in a dedicated headrest. Participants rested for $10 \mathrm{~min}$ before the tracer was injected at a mean dose of $197 \mathrm{MBq}$ of ${ }^{18} \mathrm{~F}-\mathrm{FDG}$ for patients and $4 \mathrm{MBq} / \mathrm{kg}$ of body weight for control subjects. The images were obtained with the Discovery 690 PET/CT device (GE Healthcare) with a dynamic acquisition protocol of sixty 1-min frames. Each frame had 47 slices $(3.3 \mathrm{~mm})$ and a display field of view of $250 \times$ $250 \mathrm{~mm}$ (matrix, $256 \times 256$ pixels). A CT-derived attenuation map was applied, and the data were iteratively reconstructed using time-of-flight ordered-subset expectation maximizations. The data from 45 to $60 \mathrm{~min}$ were summed and used for analysis. Images were quantified as SUVs, calculated as radioactivity concentration per gram of tissue in predefined volumes of interest relative to injected dose per $\mathrm{kg}$ of body weight.

\section{PET Image Analysis}

Segmentation. We placed 3-dimensional masks around the cerebral and cerebellar hemispheres, both ipsilateral and contralateral to the tumor, using the dedicated software ROVER (ABX) to segment the volumes of interest. We performed manual correction when a mask clearly included elements outside the brain parenchyma, for example, parts of the skull or the optic nerve.

We first tested 3 different cutoff thresholds for the detection of diaschisis: a top-down procedure with a threshold of either 2 SUVs (2T) or 3 SUVs (3T) below the $\mathrm{SUV}_{\max }$ of the cerebellum or cerebrum, respectively (thus representing the brain parenchyma and neurons with the highest level of ${ }^{18} \mathrm{~F}-\mathrm{FDG}$ accumulation, i.e., the index of functionality); a $40 \%$ iterative thresholding algorithm, with either local or global voxel analysis; and a bottom-up procedure with a threshold of $0.5 \mathrm{SUV}$, 2.0 SUV, 3.5 SUV, 4 SUV, or $50 \%$ below the $\mathrm{SUV}_{\max }$. We compared the 3 methods with respect to total hemispheric glucose metabolic ratio (THGr) and visual assessment of diaschisis, and also tested the level of statistical significance using the Kruskal-Wallis equality-of-populations rank test. The top-down $3 \mathrm{~T}$ and $2 \mathrm{~T}$ approach was found to be most appropriate for quantifying diaschisis in the cerebrum and cerebellum and included ROVER's built-in partial-volume-correction feature (11).

Individual baseline masks were reused on follow-up PET scans with the software's fixed coregistration procedure. This coregistration procedure was compared against individual placement of the masks.

\section{Interhemispheric Glucose Metabolism}

Image quantification with volumetric (3D) segmentation of malignant tissue is referred to as total lesion glycolysis (12), as first introduced by Larson et al. (13). However, gliomas are known to infiltrate healthy brain tissue and potentially induce regional diaschisis, and we previously observed depressed glucose metabolism in the entire ipsilateral cerebral hemisphere and contralateral cerebellar hemisphere in glioma patients. We thus included nonmalignant tissue in the volume of interest and delineated the entire cerebral hemisphere. We refer to the ${ }^{18} \mathrm{~F}$-FDG accumulation in the segmented volumes of interest as total hemispheric glucose metabolism (THG), which are the products of the segmented metabolic volume and the $\mathrm{SUV}_{\text {mean }}$ in the volume. THG is thus a metabolically segmented parameter, including both malignant and nonmalignant tissue and both white and gray matter.

The hemispheres of likely diaschisis, that is, the ipsilateral cerebral hemisphere of the supratentorial tumor and the contralateral cerebellar hemisphere, were normalized to the respective contralateral hemispheres, providing THG ratio indices (THGr) for the cerebrum, THGr(Ce), and cerebellum, $\operatorname{THGr}(\mathrm{Cb})$. For assessment of cerebrocerebellar diaschisis, we constructed a combined THGr index, with cutoff points provided from the area under the curve (AUC) of the receiver-operatingcharacteristic (ROC) analyses of $\operatorname{THGr}(\mathrm{Ce})$ and $\mathrm{THGr}(\mathrm{Cb})$.

\section{Visual Analysis of PET Images}

The PET images were visually assessed by a nuclear medicine physician experienced in neuroimaging and masked to the clinical outcome and the result of the quantitative analysis. Each ${ }^{18} \mathrm{~F}-\mathrm{FDG}$ image was assessed using the following scale: cerebrum: $0=$ normal (symmetric), $1=$ probably normal, 2 = unsure, 3 = probably diaschisis (hypometabolism in 2 lobes in the same hemisphere), $4=$ diaschisis (hypometabolism in 3 lobes), and $5=$ severe diaschisis (hypometabolism in 4 lobes); cerebellum: $0=$ normal (symmetric), 1 = probably normal, 2 = unsure, $3=$ probably diaschisis, and $4=$ diaschisis. (hypometabolism in the whole hemisphere).

\section{Statistical Analysis}

Survival time was calculated from the day of surgery. The KruskalWallis test was used to identify statistically significant differences of $\mathrm{THGr}(\mathrm{Ce})$ and $\mathrm{THGr}(\mathrm{Cb})$ between the participant groups. ROC analysis was used to investigate THGr as a possible predictor of survival less than 1 y. AUC of ROC was estimated, and the optimal threshold for maximizing classification was found using the point on the ROC curve closest to the $(0,1)$ corner of the ROC graph $(14)$. The optimized ROC thresholds for both cerebrum and cerebellum were applied in a $4 \times 4$ table, and the value from each set of PET images was plotted below or above the corresponding threshold. The interobserver variability of 2 interpreters for the quantitative analysis was investigated using the coefficient of variation, that is, the SD divided by the average of values obtained by the 3 observers. STATA/IC 13.1 (StataCorp) was used for all statistical analyses.

\section{RESULTS}

In total, $50{ }^{18} \mathrm{~F}-\mathrm{FDG}$ image sets were collected prospectively from the 14 patients. Two patients with low-grade glioma (diffuse astrocytoma) were alive at $3 \mathrm{y}$, whereas median survival for the 12 patients with glioblastoma was 9 mo. Baseline clinical characteristics of the glioma cohort are provided in Supplemental Table 1. Calculation of cerebrocerebellar diaschisis as THGr was straightforward. Figure 1 shows cerebrocerebellar diaschisis associated with a glioblastoma, that is, diaschises in the ipsilateral cerebral hemisphere (THGr $<$ 0.62 ) and the contralateral cerebellar hemisphere (THGr $<0.84$ ).

\section{Cerebral Diaschisis}

Supplemental Table 2 shows the THGr values and results of qualitative diaschisis assessment in the cerebrum for each patient 


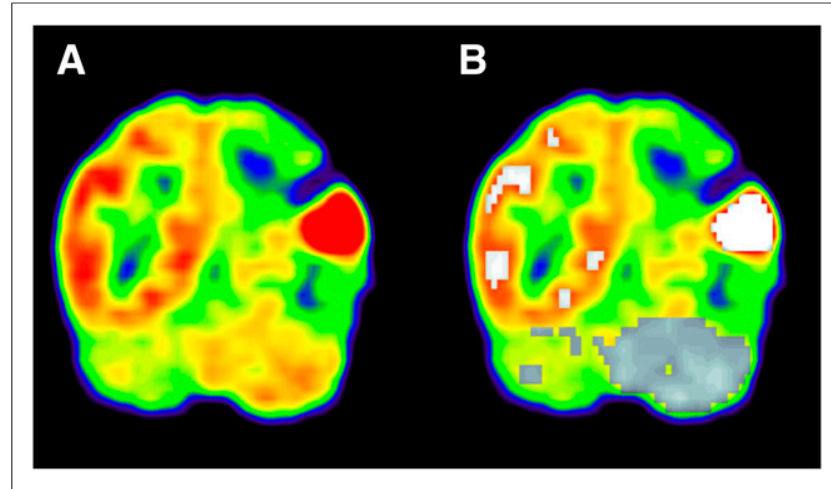

FIGURE 1. Detection of cerebrocerebellar diaschisis with ${ }^{18} \mathrm{~F}-\mathrm{FDG}$ PET. Coronal view of ${ }^{18} \mathrm{~F}-\mathrm{FDG}$ PET scan (A) and quantification of total hemispheric glucose metabolism rate (THGr) (B). Widespread diaschisis is seen in ipsilateral cerebral hemisphere and contralateral cerebellar hemisphere, to supratentorial glioblastoma. Cerebrocerebellar diaschisis was confirmed by lower THGr in cerebrum $(<0.62)$ and cerebellum $(<0.84)$. Tumor had recurred after resection, and patient died $30 \mathrm{~d}$ after scan was obtained.

over time. Figure 2 displays an example of cerebral diaschisis and segmentation with THG. Analysis of the $50{ }^{18} \mathrm{~F}-\mathrm{FDG}$ images from the glioma patients revealed a median cerebral THGr of 0.62 (range, 0.23-4.05) for patients who survived less than $1 \mathrm{y}, 0.71$ (range, $0.52-$ 1.73 ) for patients who survived $1-3 \mathrm{y}$, and 0.88 (range, $0.20-1.06$ ) for patients who survived for more than $3 \mathrm{y}$ (Fig. 3). In comparison, healthy volunteers had a median cerebral THGr of 0.98 (range, 0.791.54), which statistically was significantly higher than that of all 3 patient survival groups $(P=0.0007)$.

Glioblastoma patients who survived for more than $1 \mathrm{y}$ had a median cerebral THGr of 0.93 (range, 0.53-1.06) compared with the median of 0.62 (range, $0.23-4.05$ ) for patients with survival until $1 \mathrm{y}(P=0.046)$. The AUC of the ROC was 0.70 , and the optimal threshold of $\mathrm{THGr}(\mathrm{Ce})$ for predicting survival for more than $1 \mathrm{y}$ was 0.618 .

Cerebral THGr estimates were unusually high for 2 patients. For the patient with invasive and multifocal hypermetabolism of the entire ipsilateral hemisphere, THGr was estimated at 4.05 after resection biopsy. For the patient with (visually confirmed) recurrence who died shortly after the scan, THGr was estimated at 1.73 .

\section{Cerebellar Diaschisis}

Supplemental Table 3 shows the THGr values and results of qualitative diaschisis assessment in the cerebellum of each patient over time. Supplemental Figure 1 depicts an example of cerebellar diaschisis and segmentation with THG. Analysis of the $50{ }^{18} \mathrm{~F}-\mathrm{FDG}$ images from the glioma patients revealed a median cerebellar THGr of 0.74 (range, $0.16-1.08$ ) for patients who survived for less than $1 \mathrm{y}, 0.88$ (range, $0.52-1.01$ ) for patients who survived for $1-3 \mathrm{y}$, and 1.01 (range, 0.50-1.18) for patients who survived for more than $3 \mathrm{y}$ (Fig. 3). Healthy volunteers had a median cerebellar THGr of 0.96 (range, 0.79-1.26), which was significantly higher than those of all 3 patient survival groups $(P=0.02)$.

Glioblastoma patients who survived for more than $1 \mathrm{y}$ had a median cerebellar THGr of 0.93 (range, 0.42-1.08) compared with a median of 0.78 (range, 0.16-1.01) for patients with survival below $1 \mathrm{y}$. The AUC of the ROC was 0.77, and the optimal threshold of $\mathrm{THGr}(\mathrm{Cb})$ for predicting survival for more than $1 \mathrm{y}$ was 0.836 .

Cerebellar diaschisis typically involved the cerebellar hemisphere contralateral to the tumor. However, 1 patient (patient 7) had consecutive cerebellar diaschisis ipsilateral to the glioblastoma, which was located in the occipital lobe above the cerebellar tentorium. As we wanted to calculate the impact of diaschisis using the diaschitic hemisphere as reference point, we switched the cerebellar hemisphere of indexation for this patient.

\section{Comparison of THGr Quantification and Qualitative} Assessment

On visual examination, we detected cerebral diaschisis in $78 \%$ (39/50) of patient scans and cerebellar diaschisis in $68 \%(34 / 50)$ (Supplemental Tables 2 and 3). Cerebrocerebellar diaschisis was seen in all of the 32 PET scans of glioblastomas obtained less than $1 \mathrm{y}$ before death and in $42 \%(5 / 12)$ of the ${ }^{18} \mathrm{~F}$-FDG PET scans obtained more than $1 \mathrm{y}$ before death (including low-grade gliomas).

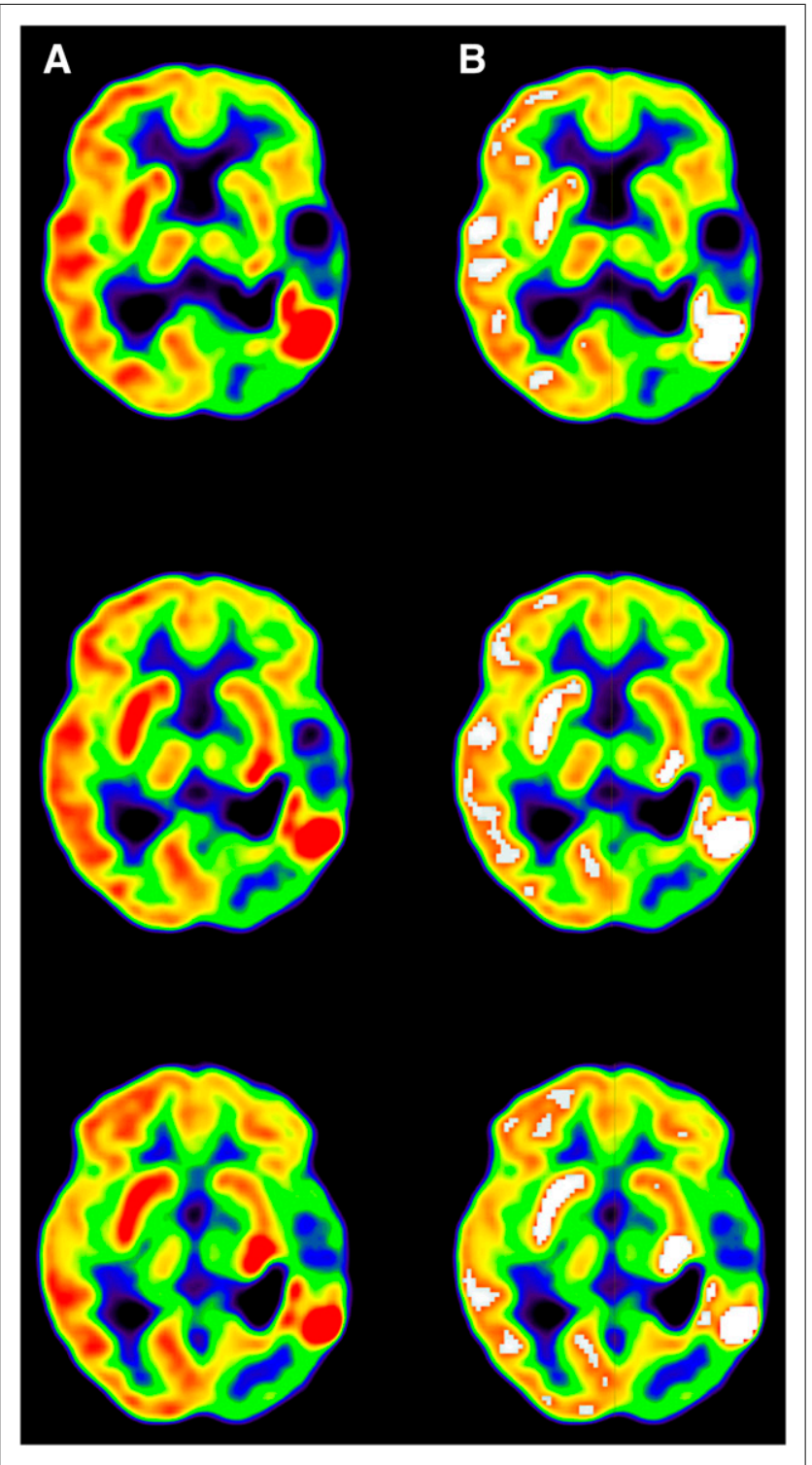

FIGURE 2. Quantification of diaschisis in cerebrum of patient with glioblastoma. ${ }^{18} \mathrm{~F}-\mathrm{FDG}$ PET scan and 3 consecutive transaxial images. Glioblastoma was located in the parietal lobe. (A) Diaschisis seen in ipsilateral frontal lobe, reaching back to occipital lobe and likely also basalganglia and thalamus. (B) THGr in diaschitic cerebral hemisphere was 0.38 , which was significantly lower than in healthy controls. 


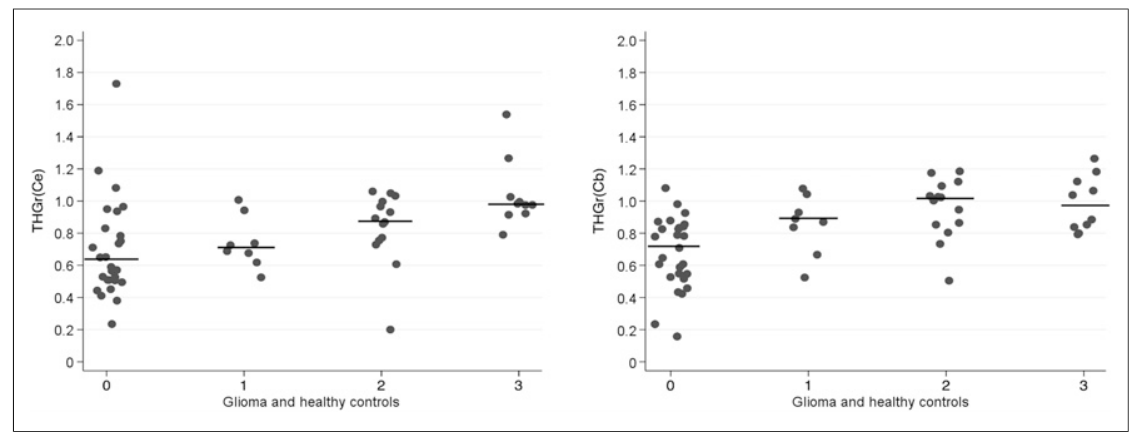

FIGURE 3. Scatterplot of THGr in cerebrum (Ce, left) and cerebellum (Cb, right) in 14 glioma patients and 10 controls. $0=$ patients surviving $<1$ y ( 8 glioblastomas), $1=$ patients surviving 1-3 y (3 glioblastomas), 2 = patients surviving $>3$ y (1 glioblastoma, 2 low-grade gliomas), $3=$ healthy controls. Gray bars display median values.

Cerebral diaschisis was seen in $94 \%$ (16/17) of cases in which cerebral THGr was low (i.e., less than 0.62), and cerebellar diaschisis was seen in $96 \%(25 / 26)$ of cases in which cerebellar THGr was low (i.e., less than 0.84).

\section{Prediction by Combining THGr for Forebrain and Hindbrain}

For initial differentiation between glioma brain and healthy brain, ROC analysis revealed optimal thresholds of $\mathrm{THGr}(\mathrm{Ce})$ at 0.91 and $\mathrm{THGr}(\mathrm{Cb})$ at 0.79 . THGr estimates below these thresholds in both the cerebrum and the cerebellum gave $100 \%$ sensitivity and $41 \%$ specificity of differentiating a patient with glioma from a healthy volunteer.

For prognosis of survival for more than $1 \mathrm{y}$ after the ${ }^{18} \mathrm{~F}$-FDG PET scan, in glioblastoma patients, the AUC of ROC revealed thresholds of $\operatorname{THGr}(\mathrm{Ce})$ at 0.62 and $\operatorname{THGr}(\mathrm{Cb})$ at 0.84 . When neither $\mathrm{THGr}(\mathrm{Ce})$ value nor $\operatorname{THGr}(\mathrm{Cb})$ value reached the threshold, the combined diaschisis measures had $100 \%$ positive predictive value. When only 1 value failed to reach the threshold, the measure had a $75 \%$ positive predictive value, and when both test values exceeded the thresholds, the measures had a $79 \%$ negative predictive value for survival below or above $1 \mathrm{y}$ for the glioblastoma patients. A positive combined diaschisis test provided $88 \%$ sensitivity and $73 \%$ specificity for survival below or above $1 \mathrm{y}$ after the PET imaging. Table 1 lists the results of the AUC of ROC thresholds used in the combined diaschisis test.

\section{Interobserver Variability and Coregistration Follow-up Images}

The median coefficients of variation for estimates by 2 independent observers were $3.3 \%$ for $\operatorname{THGr}(\mathrm{Ce})$ (interquartile range, $1.9 \%-4.2 \%$ ) and $5.9 \%$ for $\mathrm{THGR}(\mathrm{Cb})$ (interquartile range, $3.3 \%-14 \%$ ) (Supplemental Fig. 2). We found the automated reuse of the baseline 3D masks to the patients to be a robust and practical method for segmentation. When all time points were taken together, the mean differences between automated reuse and individual placement of the masks were 0.01 ( $95 \%$ confidence intervals $[\mathrm{CI}],-0.10$ to 0.13 ) for $\mathrm{SUV}_{\max }, 0.012(95 \% \mathrm{CI},-0.06$ to 0.08$)$ for $\mathrm{SUV}_{\text {mean }}$, 0.03 (95\% CI, -0.12 to 0.2 ) for metabolic volume, 5.77 (95\% CI, -24.7 to 36.3 ) for THG, -0.19 (95\% CI, -4.2 to 3.8 ) for partialvolume-corrected $\mathrm{SUV}_{\text {mean }}$, and $12.25(95 \% \mathrm{CI},-30.2$ to 54.7$)$ for

TABLE 1

Indexation of Combined Diaschisis: THGr for Glioblastoma Patients in Both Cerebrum and Cerebellum and Prognostic Implications

\begin{tabular}{|c|c|c|c|c|c|c|}
\hline Test interpretation & Test $^{\star}$ cerebrum & $\begin{array}{c}\text { Test }^{\star} \\
\text { cerebellum }\end{array}$ & $\begin{array}{l}\text { Short } \\
\text { survival } \\
(<1 \mathrm{y})^{\dagger}\end{array}$ & $\begin{array}{l}\text { Longer } \\
\text { survival } \\
(>1 \mathrm{y})\end{array}$ & Total & $\begin{array}{l}\text { Prognostic } \\
\text { implication }\end{array}$ \\
\hline Negative, both tests exceeded threshold & + & + & 3 & 11 & 14 & $\begin{array}{l}\text { Negative predictive } \\
\text { value, } 79 \%\end{array}$ \\
\hline \multicolumn{7}{|l|}{ Positive } \\
\hline \multirow[t]{2}{*}{ One test below threshold } & - & + & 3 & 1 & 4 & $\begin{array}{l}\text { Positive predictive } \\
\text { value, } 75 \%\end{array}$ \\
\hline & + & - & 9 & 3 & 12 & \\
\hline Both tests below threshold & - & - & 11 & 0 & 11 & $\begin{array}{l}\text { Positive predictive } \\
\text { value, } 100 \%\end{array}$ \\
\hline Total & & & 26 & 15 & 41 & \\
\hline Prognostic implication & & & $\begin{array}{c}\text { Sensitivity, } \\
88 \%\end{array}$ & $\begin{array}{l}\text { Specificity, } \\
73 \%\end{array}$ & & \\
\hline
\end{tabular}

${ }^{*}$ Cutoff points for $\operatorname{THGr}(\mathrm{Ce})$ and $\mathrm{THGr}(\mathrm{Cb})$, provided from AUC ROC analysis, respectively; dichotomization for cerebral THGr, $\geq 0.62$ and $\leq-0.62$. Dichotomization for $\operatorname{THGr}(\mathrm{Cb}), \geq 0.84$ and $\leq-0.84$.

${ }^{\dagger}$ PET scan obtained less than or more than 12 mo before death.

Cerebrocerebellar diaschisis was assessed by combined THGr index using cutoff points derived from AUC of ROC analysis of THGr (Ce) and $\mathrm{THGr}(\mathrm{Cb})$. In patients with glioblastoma, low $\mathrm{THGr}$ in both regions $(\mathrm{Ce}<0.62$ and $\mathrm{Cb}<0.84)$ had $100 \%$ positive predictive value for glioblastoma survival $<1$ y after ${ }^{18} \mathrm{~F}$-FDG PET scan. If both $\mathrm{THGr}(\mathrm{Ce})$ and $\mathrm{THGr}(\mathrm{Cb})$ were above thresholds, negative predictive value was $78.6 \%$ for survival with glioblastoma of more than $1 \mathrm{y}$. 
partial-volume-corrected THG. None of these differences was statistically significant $(P>0.05)$ (Supplemental Table 4). We also compared reuse of baseline masks stratified to any specific treatment intervention, that is, after operation or chemotherapy; no statistically significant difference was found either ( $P$ value, $0.055-0.96)$. Automated reuse of the $3 \mathrm{D}$ masks allowed excellent delineation of the cerebral and cerebellar structures for visual assessment.

\section{DISCUSSION}

The study results showed that estimation of THGr was straightforward and meaningfully could quantify diaschisis in the cerebrum and cerebellum of patients with glioma. Quantitative and visual detection of diaschisis was associated with shorter survival. THGr estimates had low interobserver variability despite differences of patient treatments with neurosurgery, radiotherapy, and chemotherapy.

\section{Diaschisis in Patients with Glioblastoma}

The mean age $(63 \mathrm{y})$ and median survival $(9 \mathrm{mo})$ for the glioblastoma patients were similar to those reported in the literature (63.7 y and $9.9 \mathrm{mo}$ ) (15). Glioblastoma patients who survived less than $1 \mathrm{y}$ had low THGr in most of their cerebral and cerebellar images. Glioblastoma patients surviving for more than $1 \mathrm{y}$ had a few images with THGr below ROC threshold but not at the same time in both the cerebrum and the cerebellum. The presence of cerebrocerebellar diaschisis detected with the combined diaschisis test thus had a $100 \%$ positive predictive value of survival for less than $1 \mathrm{y}$. Our findings suggest that an unusually high $\mathrm{THGr}$ value may also indicate severe disease (e.g., widespread invasive disease and very short survival), but this is based on only 2 patients with high values. Both these patients either had a biopsy performed (patient 6) or had a partial removal (patient 4); the neurosurgical intervention might be interesting to elaborate further with THGr in patients with glioma and the correlation to diaschisis.

\section{Low-Grade Gliomas}

The 2 patients with low-grade gliomas did not display any significant cerebrocerebellar diaschisis during the follow-up. Although one third of the 9 images showed low cerebral THGr, the cerebrocerebellar pathways probably were not significantly affected by the neoplasms, because the cerebellar THGr values exceeded the ROC thresholds. The patient with the lowest cerebral THGr values had chronic hearing loss originating in the ipsilateral hemisphere after resection as well as aggressive Jackson epileptic seizures, and this was the only patient with low-grade glioma who experienced cancer recurrence. In the light of the THGr results presented for low-grade gliomas, our hypothesis is that if a lowgrade glioma presents with a positive combined diaschisis test, this might indicate progression to glioblastoma/progressive damage to the neuronal connections.

\section{Strengths and Weaknesses}

This method of quantifying diaschisis improves the understanding of some poorly understood pathophysiologic mechanisms in the brain, which may help to improve management strategies for patients with glioma. The study had a small sample size, was exploratory in nature, and time between scans and treatment was heterogeneous. Hence, confounding factors such as age, sex, and treatment intervention were not suitable for a multivariate analysis. However, we systematically and prospectively collected 50 ${ }^{18} \mathrm{~F}$-FDG images during the course of treatment of patients for glioma that constitutes a unique material in the literature. The longitudinal study design enabled us to investigate the presence of diaschisis at 3-6 time points during a time period of between 3 mo and more than a year, which validated the reproducibility of the method across heterogeneous treatment interventions. The combined estimation of THGr values in the ipsilateral cerebrum and the contralateral cerebellum provided a way of quantifying cerebrocerebellar diaschisis that would allow comparison of results between institutions, with less interference from misinterpretation of SUV, and differences in tracer dose, device brand, and acquisition times (16).

The method presented here may be relevant also for other brain diseases. ${ }^{18} \mathrm{~F}$-FDG imaging has suggested the presence of diaschisis in multiple sclerosis (17), Lyme disease (18), thalamocortical lesions (5), traumatic diffuse brain injury (19), and dementia (20). Baron et al. found that a cerebral hemispheric ratio below 0.85 in thalamocortical lesions consistently was associated with cognitive impairment (5). Psychologic disorders can also display decreased regional or global glucose metabolism, which can be speculated to also be caused by diaschisis, for example, chronic fatigue syndrome (21), posttraumatic stress disorder (22), major depressive disorder (23), and chronic depression syndromes (24).

Moreover, diaschisis has been suggested to represent a reversible neurologic phenomenon by Sobesky et al. (25). They found that reversal of diaschisis along the treatment trajectory was associated with better clinical outcome in stroke patients. In this respect, the THGr method presented might be a means of monitoring quantitatively treatment effects in stroke patients or other brain diseases with diaschisis.

In the glioma material, a $3 \mathrm{~T}$ and $2 \mathrm{~T}$ top-down cutoff provided the best prognostic information by revealing the extent of diaschisis. However, other brain diseases may require different algorithms, and the specific cutoff points require validation by comparison with a suitable reference. Glioblastomas normally are recognized by their hypermetabolism (26). However, we observed that some or all of the ipsilateral hemisphere of glioblastoma patients had lower glucose metabolism than the contralateral hemisphere. This observation is sensitive to the potential effects of edema surrounding the tumor, which may create a false impression of hypometabolism as demonstrated by Alavi et al. (27). Future studies of diaschisis in gliomas will need to take this into account by supplementing with information obtained by CT or preferably MRI.

\section{CONCLUSION}

The THGr measures demonstrated cerebral and cerebellar diaschisis in patients with glioma. ROC analysis demonstrated cerebrocerebellar diaschisis ratios to have significant positive predictive value for survival below $1 \mathrm{y}$ and negative predictive value for survival above $1 \mathrm{y}$. The THGr method allows comparison of data obtained at different institutions and is now open for further validation in gliomas and other cerebral diseases.

\section{DISCLOSURE}

No potential conflict of interest relevant to this article was reported.

\section{ACKNOWLEDGMENT}

We thank Asbjorn Hrobjartsson for his contributions. 


\section{REFERENCES}

1. VonMonakow C. Die Localization im Grosshirn und der Abbau der Funktion Durch Korticale Herde. Wiesbaden, Germany: JF Bergmann; 1914.

2. Azevedo FA, Carvalho LR, Grinberg LT, et al. Equal numbers of neuronal and nonneuronal cells make the human brain an isometrically scaled-up primate brain. J Comp Neurol. 2009;513:532-541.

3. Carrera E, Tononi G. Diaschisis: past, present, future. Brain. 2014;137:2408-2422.

4. Hess S, Jakobsen NU, Brøndserud M, Segtnan EA, Schifter J. FDG-PET/CT for systemic infections. Curr Mol Imaging. 2014;3:182-190.

5. Baron JC, Levasseur M, Mazoyer B, et al. Thalamocortical diaschisis: positron emission tomography in humans. J Neurol Neurosurg Psychiatry. 1992;55:935-942.

6. Alavi A, Mirot A, Newberg A, et al. Fluorine-18-FDG evaluation of crossed cerebellar diaschisis in head injury. J Nucl Med. 1997;38:1717-1720.

7. Agarwal KK, Tripathi M, Karunanithi S, Das CJ, Suri V, Nalwa A. 3T. Rev Esp Med Nucl Imagen Mol. 2014;33:397-398.

8. Al-Faham Z, Zein RK, Wong CY. ${ }^{18}$ F-FDG PET assessment of Lewy body dementia with cerebellar diaschisis. J Nucl Med Technol. 2014;42:306-307.

9. Agrawal KL, Mittal BR, Bhattacharya A, Khandelwal N, Prabhakar S. Crossed cerebellar diaschisis on F-18 FDG PET/CT. Indian J Nucl Med. 2011;26:102-103.

10. Fedorov A, Beichel R, Kalpathy-Cramer J, et al. 3D Slicer as an image computing platform for the Quantitative Imaging Network. Magn Reson Imaging. 2012;30:1323-1341.

11. Hess S, Rakheja R, Friedman K, et al. A brief overview of novel approaches to FDG PET imaging and quantification. Clin Transl Imaging. 2014;2:187-198.

12. Basu S, Zaidi H, Salavati A, Hess S, Carlsen PF, Alavi A. FDG PET/CT methodology for evaluation of treatment response in lymphoma: from "graded visual analysis" and "semiquantitative SUVmax" to global disease burden assessment. Eur J Nucl Med Mol Imaging. 2014;41:2158-2160.

13. Larson SM, Erdi Y, Akhurst T, et al. Tumor treatment response based on visual and quantitative changes in global tumor glycolysis using PET-FDG imaging: the visual response score and the change in total lesion glycolysis. Clin Positron Imaging. 1999;2:159-171.

14. Coffin M, Sukhatme S. Receiver operating characteristic studies and measurement errors. Biometrics. 1997;53:823-837.

15. Helseth R, Helseth E, Johannesen TB, et al. Overall survival, prognostic factors, and repeated surgery in a consecutive series of 516 patients with glioblastoma multiforme. Acta Neurol Scand. 2010;122:159-167.
16. Keyes JW Jr. SUV: standard uptake or silly useless value? J Nucl Med. 1995;36:1836-1839.

17. Bakshi R, Miletich RS, Kinkel PR, Emmet ML, Kinkel WR. High-resolution fluorodeoxyglucose positron emission tomography shows both global and regional cerebral hypometabolism in multiple sclerosis. J Neuroimaging. 1998;8:228-234.

18. Newberg A, Hassan A, Alavi A. Cerebral metabolic changes associated with Lyme disease. Nucl Med Commun. 2002;23:773-777.

19. Nakayama N, Okumura A, Shinoda J, Nakashima T, Iwama T. Relationship between regional cerebral metabolism and consciousness disturbance in traumatic diffuse brain injury without large focal lesions: an FDG-PET study with statistical parametric mapping analysis. J Neurol Neurosurg Psychiatry. 2006;77:856-862.

20. Schöll M, Damian A, Engler H. Fluorodeoxyglucose PET in neurology and psychiatry. PET Clin. 2014;9:371-390.

21. Siessmeier T, Nix WA, Hardt J, Schreckenberger M, Egle UT, Bartenstein P. Observer independent analysis of cerebral glucose metabolism in patients with chronic fatigue syndrome. J Neurol Neurosurg Psychiatry. 2003;74: 922-928.

22. Tanev K. Neuroimaging and neurocircuitry in post-traumatic stress disorder: what is currently known? Curr Psychiatry Rep. 2003;5:369-383.

23. Su L, Cai Y, Xu Y, Dutt A, Shi S, Bramon E. Cerebral metabolism in major depressive disorder: a voxel-based meta-analysis of positron emission tomography studies. BMC Psychiatry. 2014;14:321-329.

24. Song SH, Kim IJ, Kim SJ, Kwak IS, Kim YK. Cerebral glucose metabolism abnormalities in patients with major depressive symptoms in pre-dialytic chronic kidney disease: statistical parametric mapping analysis of F-18-FDG PET, a preliminary study. Psychiatry Clin Neurosci. 2008;62:554-561.

25. Sobesky J, Thiel A, Ghaemi M, et al. Crossed cerebellar diaschisis in acute human stroke: a PET study of serial changes and response to supratentorial reperfusion. J Cereb Blood Flow Metab. 2005;25:1685-1691.

26. Segtnan EA, Hess S, Grupe P, Hoilund-Carlsen PF. ${ }^{18} \mathrm{~F}$-fluorodeoxyglucose PET/computed tomography for primary brain tumors. PET Clin. 2015;10: 59-73.

27. Pourdehnad M, Basu S, Duarte P, et al. Reduced grey matter metabolism due to white matter edema allows optimal assessment of brain tumors on ${ }^{18} \mathrm{~F}-\mathrm{FDG}$-PET. Hell J Nucl Med. 2011;14:219-223. 